\title{
Atomistic model for nearly quantitative simulations of Langmuir monolayers
}

\author{
Javanainen, Matti
}

2018-02-20

Javanainen, M , Lamberg , A , Cwiklik , L , Vattulainen , I T \& Ollila , S 2018 , ' Atomistic model for nearly quantitative simulations of Langmuir monolayers ' , Langmuir , vol. 34 , no. pÿ7 , pp. 25652572 . https://doi.org/10.1021/acs.langmuir.7b02855

http://hdl.handle.net/10138/321527

https://doi.org/10.1021/acs.langmuir.7b02855

unspecified

acceptedVersion

Downloaded from Helda, University of Helsinki institutional repository.

This is an electronic reprint of the original article.

This reprint may differ from the original in pagination and typographic detail.

Please cite the original version. 


\title{
Atomistic Model for Near-Quantitative Simulations of Langmuir Monolayers
}

\author{
Matti Javanainen, ${ }^{, \dagger,+}$ Antti Lamberg, Lukasz Cwiklik, ${ }^{\S, \|}$ Ilpo Vattulainen, ${ }^{\ddagger}, \dagger, \perp$ \\ and O. H. Samuli Ollila*,\|,\# \\ $\dagger$ Laboratory of Physics, Tampere University of Technology, Tampere, Finland \\ $\ddagger$ Department of Physics, University of Helsinki, Helsinki, Finland \\ ฯ Unaffiliated \\ $\S J$. Heyrovsky Institute of Physical Chemistry, Academy of Sciences of the Czech Republic, \\ Prague 8, Czech Republic \\ || Institute of Organic Chemistry and Biochemistry, Czech Academy of Sciences, Prague 6, \\ Czech Republic \\ $\perp$ MEMPHYS - Center for Biomembrane Physics \\ \#Institute of Biotechnology, University of Helsinki, Helsinki, Finland \\ E-mail: matti.javanainen@tut.fi; samuli.ollila@helsinki.fi
}

\begin{abstract}
Lung surfactant and tear film lipid layer are examples of biologically relevant macromolecular structures found at the air-water interface. Due to their complexity, they are often studied in terms of simplified lipid layers, the simplest example being a Langmuir monolayer. Given the profound biological significance of these lipid assemblies, there is a need to understand their structure and dynamics in the nanoscale, yet there are not many techniques able to provide this information. Atomistic molecular dynamics simulations would be a tool fit for this purpose, however the simulation models suggested
\end{abstract}


until now have been qualitative instead of quantitative. This limitation has mainly stemmed from the challenge to correctly describe the surface tension of water with simulation parameters compatible with other biomolecules. In this work, we show that this limitation can be overcome by using the recently introduced four-point OPC water model, whose surface tension for water is demonstrated to be quantitatively consistent with experimental data, and which is also shown to be compatible with the commonly employed lipid models. We further establish that the approach of combining the OPC four-point water model with the CHARMM36 lipid force field provides near-quantitative agreement with experiments for the surface pressure-area isotherm for POPC and DPPC monolayers, including also the experimentally observed phase coexistence in a DPPC monolayer. The simulation models reported in this work pave the way for near-quantitative atomistic studies of lipid-rich biological structures at air-water interfaces.

\section{Introduction}

Lipid monolayers at the air-water interface are important model systems often used to study, e.g., lung surfactant function, ${ }^{1}$ tear film lipid layer structure, ${ }^{2}$ and membrane protein insertion. $^{3}$

While the thermodynamics of lipid layers at the air-water interface is understood quite well, the molecular-level details of phenomena taking place at these interfaces are difficult to access experimentally. ${ }^{4}$ This is largely due to the nanometer resolution required to explore the structure and the dynamics of soft interfaces that are in constant motion due to thermal fluctuations. This makes atomistic and coarse-grained (CG) molecular dynamics (MD) simulations often the method of choice to investigate conditions that are exactly

of this kind. They have been used, e.g., to study phospholipid monolayers, ${ }^{5,6}$ pulmonary surfactant layers, ${ }^{6,7}$ and tear film lipid ${ }^{2,8-12}$ structures to clarify monolayer phase behavior and dynamics, ${ }^{13-16}$ the collapse transition, ${ }^{17-19}$ pore formation, ${ }^{20,21}$ as well as interactions of 
lipids with proteins ${ }^{22}$ and nanoparticles. ${ }^{21,23}$

Quantitative studies of these phenomena through MD simulations are, however, quite impossible given the fact that the simulation models used in these studies seriously underestimate the surface tension of water. While the widely employed atomistic biomolecular force fields Slipids, ${ }^{24}$ OPLS-AA, ${ }^{25}$ Lipid14, ${ }^{26}$ and CHARMM36 ${ }^{27}$ are quite successful in describing complex water-embedded lipid and lipid-protein systems such as biomembranes, their applicability at the air-water interface is compromised by the quality of the water model. For instance, the TIP3P water model ${ }^{28}$ or its derivatives, which are typically used in conjunction with the above-mentioned biomolecular force fields, yields a value of $\sim 50 \mathrm{mN} / \mathrm{m}^{29,30}$ for the surface tension of water at room temperature, while the experimental value is $72 \mathrm{mN} / \mathrm{m} .{ }^{31}$ In CG simulations the situation can be even worse as is exemplified by the surface tension of about $30 \mathrm{mN} / \mathrm{m}$ given by the commonly used Martini model. ${ }^{32}$ Consequently, most simulation models cannot reproduce experimental pressure-area isotherms ${ }^{5,13,33-37}$ with quantitative accuracy, and phenomena such as phase transitions, monolayer collapse, and pore formation do not take place at the correct surface tension values. ${ }^{13,33}$

To overcome these issues, the simulation results are often interpreted by assuming that the pressure-area isotherm can be shifted along the pressure axis by a constant to compensate for the effect of an incorrect surface tension. ${ }^{5,6,13}$ However, the error arising from the incorrect surface tension cannot be corrected through the addition of a constant factor ${ }^{33,38}$ and the isotherms reported in, e.g., refs. 34 and 35 cannot reproduce experimental data with a constant shifting factor (see Fig. S8 in SI). Water models with a surface tension that is too low do underestimate the energy cost of exposing the air-water interface in simulations, which leads to the formation of pores or nonphysical (negative) surface pressures at lipid densities where the monolayer should be in the liquid-condensed $\left(\mathrm{L}_{\mathrm{C}}\right)$ or the liquid-expanded $\left(\mathrm{L}_{\mathrm{E}}\right)$ phase. ${ }^{33,34,37,39}$ On the other hand, phase transitions and monolayer collapse take place at too large surface pressures. For example, the Martini model gives $\mathrm{L}_{\mathrm{C}} / \mathrm{L}_{\mathrm{E}}$ phase coexistence for DPPC with surface pressures that correspond to the equilibrium collapse pressure in 
experiments, and the monolayer remains stable even with negative surface tension values. ${ }^{13}$ Given all these issues, it is clear that there is a need for water models that could provide a correct surface tension and thereby render realistic MD simulations of Langmuir monolayers possible. ${ }^{33,37,39}$

While experimental pressure-area isotherms have been reproduced in certain CG models with a correct surface tension of water, ${ }^{39}$ for atomistic simulations the task is more complicated. The typically employed atomistic water models reproduce the experimentally observed surface tension only with large cut-off distances for the Lennard-Jones (LJ) interaction. ${ }^{40}$ This is exemplified by the TIP4P/2005 force field, ${ }^{41}$ which provides the most optimal surface tension among the rigid 3-point and 4-point water models, ${ }^{29,30,42}$ yet it requires a large LJ cut-off distance above $2 \mathrm{~nm}$ (or the use of a long-range Ewald summation algorithm for the LJ interaction ${ }^{43,44}$ ) to gain reasonable agreement with experiments. ${ }^{40,45}$ Since this cut-off distance is significantly larger than the values commonly employed in lipid models $(1.0-1.4 \mathrm{~nm}),{ }^{24-27}$ it leads to other problems: increasing the LJ cut-off distance above the values used in lipid force field parametrization leads to artificial behavior in describing the given lipid systems. ${ }^{46,47}$ The pressure-area isotherm cannot therefore be reproduced by just increasing the LJ cut-off distance in order to match the surface tension of water to a more realistic value, because this would compromise the quality of the simulation model of the lipid monolayer. ${ }^{36,37}$ Additionally, while one may be tempted to use isotropic dispersion corrections $^{48}$ to overcome this issue, one has to keep in mind that they are by design not suitable for heterogeneous systems such as interfaces. Furthermore, adjustment of this kind does not result in the correct balance of forces at the interfaces. An anisotropic dispersion correction has been recently introduced for planar surfaces, ${ }^{49}$ but it is not yet available in any simulation software and might require a reparametrization of the existing force fields.

In this work, we show that the recently suggested 4-point "Optimal Point Charge" (OPC4) water model ${ }^{50}$ provides a solution to this challenging problem. As has been shown before, ${ }^{50}$ OPC4 accurately describes the bulk properties of water even with short LJ cut-off values. In 
this work, we first show that the surface tension of water can also be reproduced with the OPC4 model by using LJ cut-off lengths compatible with the CHARMM36 lipid model. ${ }^{27}$ Second, using lipid bilayers as our benchmark, we show that OPC4 can be successfully combined with the CHARMM36 lipid model. ${ }^{27}$ Third, we extend these CHARMM36/OPC4 simulations to lipid monolayers at the air-water interface: the combination of these two force fields quantitatively reproduces the experimental surface pressure-area isotherms for 1-palmitoyl-2-oleoylphosphatidylcholine (POPC) and dipalmitoylphosphatidylcholine (DPPC) monolayers, including also the coexistence region of the $\mathrm{L}_{C}$ and $\mathrm{L}_{\mathrm{E}}$ phases. Similar quantitative agreement with experimental data has not been achieved previously. Finally, we show that in

addition to CHARMM36, the Slipids force field ${ }^{24}$ can also be successfully combined with the OPC4 water model.

The approach reported here paves the way for quantitative atomistic studies of lipid-rich biological structures at air-water interfaces. All the simulation data and related files of the model systems are publicly available for further use (see Section S3).

\section{Simulation Models and Methods}

\section{Bulk Water and the Air-Water Interface}

We first simulated a pure air-water interface using the 3-point $\mathrm{OPC}^{51}$ (OPC3) and 4-point $\mathrm{OPC}^{50}$ (OPC4) water models to evaluate the performance of the models with different cut-off lengths. To this end, a cubic box with an edge length of $4 \mathrm{~nm}$ was first filled with 2228 water molecules. After energy minimization, the box vectors in the $z$-direction were extended to $8 \mathrm{~nm}$, thereby creating a water slab which had two interfaces with air (vacuum). These systems were then simulated for $5 \mathrm{~ns}$ at constant volume and at three different temperatures (298, 323, and $348 \mathrm{~K})$ using parameters mimicking as closely as possible those employed in the original publication, ${ }^{50}$ referred to as the "OPC parameter set" from here on (see Table S1 for details). In addition to the original LJ cut-off of $0.8 \mathrm{~nm}$, values up to $1.4 \mathrm{~nm}$ were tested, 
both with and without a dispersion correction ${ }^{48}$ applied to energy and pressure. Furthermore, the simulations were repeated with LJ-PME ${ }^{47,52}$ (Lennard-Jones particle mesh Ewald).

Surface tension $(\gamma)$ was calculated from the planar $\left(P_{L}\right)$ and normal $\left(P_{N}\right)$ components of the pressure as

$$
\gamma=\frac{1}{2}\left(P_{N}-P_{L}\right) \times L_{z}
$$

where $P_{L}=\frac{1}{2}\left(P_{x x}+P_{y y}\right)$ and $L_{z}$ is the length of the simulation box edge that is normal to the interface, and the prefactor of $1 / 2$ accounts for the two interfaces present in the system. The first 500 ps of the simulations were omitted from analyses.

Bulk water simulations were conducted to evaluate the performance of the OPC water model with the simulation parameters suggested to be used with the CHARMM36 force field with GROMACS, from now on referred to as the "CHARMM36 parameter set" (see Table S1 for details). For this purpose, a cubic box with 2228 OPC4 water molecules was simulated at $298 \mathrm{~K}$ and 1 bar for $5 \mathrm{~ns}$, the first $1 \mathrm{~ns}$ of which was omitted in analyses. Notably for compatibility with lipid models, the used parameters were the same as those employed for lipid bilayer and monolayer simulations described below, with the exception that isotropic pressure coupling was used here for bulk water. Additionally, the calculation was repeated with and without the dispersion correction, as suggested for bilayers and monolayers simulated with CHARMM36, in respective order. All simulations were run with GROMACS 5.0.x. ${ }^{53}$ Similar simulations were repeated also with the simulation parameters recommended for the Slipids model (with isotropic pressure coupling), from here on referred to as the "Slipids parameter set" (see Table S1). These simulations, detailed in Sections S1.2 and S2.1, were performed with GROMACS 4.6.x. ${ }^{54}$

Finally, the surface tension values of water $\left(\gamma_{0}\right)$ used in the calculation of the surface pressures of the monolayers (see Eq. 2) were extracted from simulations of larger air-water interface systems. These systems contained 20052 water molecules with dimensions of 
$12 \times 12 \times 22 \mathrm{~nm}^{3}$ corresponding to the size of an average monolayer simulation described below.

\section{Lipid Bilayers}

Lipid bilayer simulations were run to evaluate the quality of the CHARMM36 lipid model ${ }^{27}$ when combined with the OPC4 water model. For this case, lipid bilayers with 200 DPPC or POPC molecules were built with CHARMM-GUI ${ }^{55}$ and simulated at $323 \mathrm{~K}$ (DPPC) or 303 K (POPC) for 100 ns. Bilayers were hydrated with 9000 water molecules (45 per lipid) and simulated with OPC4. ${ }^{50}$ Similar simulations were also carried out with the TIP(S)3P (CHARMM-specific TIP3P) water model. ${ }^{28}$ The CHARMM36 parameter set was employed (see Table S1). All simulations were performed with GROMACS 5.0.x. ${ }^{53}$

Trajectories were recorded every 100 ps, and the first 10 ns of every 100 ns simulation trajectory was omitted from analysis. Density profiles were calculated using the tool g_density, and deuterium order parameters using the tool g_order, both bundled with the GROMACS simulation suite. Area per lipid (APL) was calculated by dividing the membrane area by the

number of lipids. Similar simulations were carried out using the Slipids model ${ }^{24,56}$ with the Slipids parameter set (see Table S1) using GROMACS 4.6.x ${ }^{54}$ to assess its performance with the OPC4 water model (see Sections S1.3 and S2.2).

\section{Lipid Monolayers at the Air-Water Interface}

Lipid monolayers at the air-water interface were simulated with different values for the average APL to calculate the surface pressure-area isotherms needed for comparison with experimental data. First, a system containing two POPC monolayers with a total of 512 lipids (256 per monolayer) with an average APL of $50 \AA^{2}$ and a total of 41482 water molecules was generated. This corresponds to a total of 232488 atoms. The monolayers were deposited on a water slab having an interface (in the $x y$-plane) with air (vacuum) on both sides. The box size in the $z$-direction was set to $22 \mathrm{~nm}$ to avoid unscreened electrostatic interactions through vacuum. The system was energy minimized and simulated with lipid head groups 
restrained in the $z$-dimension for $50 \mathrm{~ns}$. This was followed by a $150 \mathrm{~ns}$ simulation without restraints.

Next, monolayer structures were extracted at 50, 100, and $150 \mathrm{~ns}$ of the simulations. These structures were then each expanded during a $10 \mathrm{~ns}$ simulation to an average APL value of $130 \AA^{2}$ using the MOVINGRESTRAINT and CELL keywords in the PLUMED 2.2 package. ${ }^{57}$ From the first expansion simulation, frames corresponding to APLs of 55, 58, 61, 64, 67, 70, $78,86,94,102,110,118$, and $126 \AA^{2}$ were extracted. From the second expansion simulation, frames corresponding to APLs of 52, 60, 66, 72, 90, 106, and $122 \AA^{2}$ were extracted, and from the third expansion simulation, frames corresponding to APLs of 57,63, 69, 82, 98, and $114 \AA^{2}$ were extracted. Extracting these initial structures from three independent expansion simulations guarantees their mutual independency.

Initial frames for DPPC simulations were generated as follows: From the first POPC expansion simulations, frames corresponding to 51, 54, 57, 60, 63, 66, 69, 72, 75, 78, 86, 94, 102, and $110 \AA^{2}$ were extracted. From the second expansion simulation, the corresponding values were $53,59,65,71,77,90$, and $106 \AA^{2}$, while from the third expansion simulation systems with APLs equal to 50, 56, 62, 68, 74, 82, and $98 \AA^{2}$ were extracted. Next, POPC molecules were modified to DPPC lipids, the systems energy-minimized, briefly equilibrated, and subsequently simulated. For DPPC monolayers at areas below $50 \AA^{2}$, the DPPC monolayer at $51 \AA^{2}$, obtained as described above, was compressed using PLUMED. The frames at APLs of 45 and $48 \AA^{2}$ were then extracted. The simulation sets extracted from the three expansion simulations are named Sets 1-3, respectively.

The DPPC monolayers were simulated at both 310 and $298 \mathrm{~K}$ for at least $100 \mathrm{~ns}$. In all monolayer simulations, if the monolayer surface pressure calculated from the last $50 \mathrm{~ns}$ of the simulation had not converged, the simulations were extended in 50 ns periods until this criterion was fulfilled. The convergence was finally verified by analyzing values from separate 25 ns periods of the simulation trajectories. The results shown in Figs. S3-S5 conclude that convergence was reached in all simulations, and the lengths of the simulations are shown in 
Table S2.

The monolayer simulation parameters were identical with the bilayer ones ("CHARMM36 parameter set"), except they were performed in the NVT ensemble and the dispersion correction ${ }^{48}$ was applied to both energy and pressure (see Table S1 for details).

Monolayer surface pressure $\Pi(a)$ was obtained from Eq. 2. Surface tensions for a pure air-water interface and for an interface with the lipid monolayer were calculated with Eq. 1.

Fractions of $\mathrm{L}_{\mathrm{E}}$ and $\mathrm{L}_{\mathrm{C}}$ phases in the coexistence region were estimated using Voronoi tessellation provided by the MEMBPLUGIN tool, ${ }^{58}$ which was modified to generate output data for all lipids individually at each analyzed frame. The APL values for all lipid molecules were first collected from all analyzed simulation frames from all the simulations conducted within the coexistence plateau. Next, these APL values were used to build histograms that were fit with two Gaussian functions, and the mean values of the these distributions were considered to be characteristic for the two phases. Next, data from individual systems were fit with two Gaussians, whose mean values were fixed to those defined from the fits described above. The relative magnitudes of the prefactors of the two Gaussians were considered to represent the relative amounts of the two phases present. For APL values larger and smaller than those where phase coexistence is manifested, the double Gaussian description was found to fit the data poorly, as expected.

Simulation data and related files are available online (see Section S3). Also simulation data for monolayers with smaller box sizes and with the Slipids model, discussed in SI, have been made available (see Section S3).

\section{Monolayer Surface Pressure and Its Comparison with Experimental}

\section{Data}

In experiments, surface pressure-area isotherms are measured by compressing a given amount of surfactant deposited at an air-water interface and measuring the mechanical interfacial tension $\gamma(a)$ as a function of area per molecule $a$. The surface pressure of a monolayer is then 
defined as

$$
\Pi(a)=\gamma_{0}-\gamma(a)
$$

where $\gamma_{0}$ is the surface tension of water.

Compressing the lipid monolayer increases the repulsion between molecules, which leads to an increase in the chemical potential of the surfactant at the interface. When the chemical potential at an interface and in bulk aggregates (e.g., lamellar or micellar structures) is equal, further compression usually leads to transfer of molecules from the interface to bulk aggregates instead of an increase in surface pressure. The surface pressure at this point is called the equilibrium collapse pressure and it equals the spreading pressure of the molecules. ${ }^{59,60}$ Carefully equilibrated experiments give values of $\Pi$ close to $45 \mathrm{mN} / \mathrm{m}$ for phosphatidylcholine lipids independently of the acyl chain content. ${ }^{59,60}$

Monolayers formed by some surfactants, such as DPPC with two saturated acyl chains, can be, however, compressed to a metastable state having a surface pressure above the equilibrium collapse point. ${ }^{1}$ These states have a very low mechanical surface tension, which is believed to be important for lung functionality. ${ }^{1}$ Low mechanical surface tension does not, however, mean low energy cost for a change in area, because it is not the derivative of the free energy with respect to area, which is the thermodynamical surface tension in equilibrium.

In this work we focus on the surface pressure-area isotherms for pressures below the equilibrium collapse pressure $\left(\sim 45 \mathrm{mN} / \mathrm{m}\right.$ for phospholipids $\left.{ }^{59,60}\right)$. The simulations are carried out with significant computational resources to reach the phase coexistence region in DPPC monolayer. Even more extensive simulations would be required to determine the collapse pressure and the collapse mechanism, as well as to study realistic metastable structures above the equilibrium collapse pressure. While these studies are beyond the scope of this work, we note that the proposed combination of CHARMM36 and OPC4 models should allow quantitative simulations of these phenomena with atomistic resolution. 


\section{Results and Discussion}

\section{Water Model With Correct Surface Tension for Lipid Simulations}

First, for the OPC $3^{51}$ and $\mathrm{OPC} 4{ }^{50}$ water models, we evaluated the effect of the LJ cut-off and the use of the dispersion correction on the air-water surface tension. The comparison to experiment was made at three different temperatures $(298,323$, and $348 \mathrm{~K})$. As shown in Fig. 1, the OPC4 model reproduces the experimental values at all three temperatures, when the LJ cut-off is set to $1.2-1.4 \mathrm{~nm}$. Values smaller than this, including the $0.8 \mathrm{~nm}$ used in the original OPC4 publication, ${ }^{50}$ lead to underestimation of surface tension, whereas using LJ-PME leads to its overestimation. Interestingly, the values obtained with and without the dispersion correction (lighter and darker lines in Fig. 1) are almost identical. Meanwhile, the OPC3 water model underestimates surface tension with all tested LJ cut-off settings.

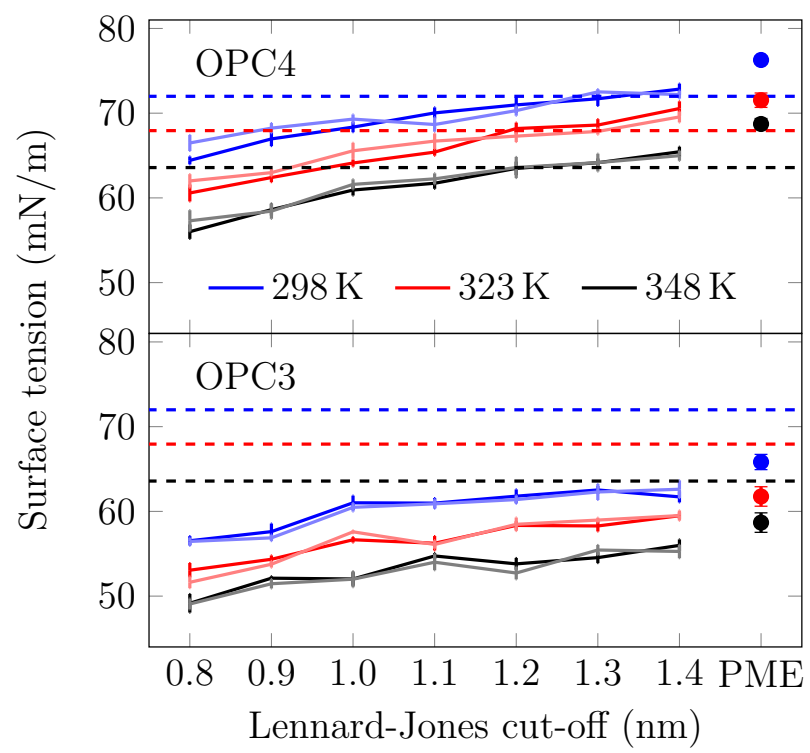

Figure 1: The air-water surface tension values calculated using different LJ cut-offs (solid lines) or the LJ-PME algorithm (points). The results with and without the dispersion correction applied to both energy and pressure are shown with lighter and darker lines, respectively. The horizontal dashed lines show experimental values.

Importantly, the OPC4 water model reproduces the surface tension of water very well with the LJ cut-off length equal to $1.2 \mathrm{~nm}$, which is used in the CHARMM36 lipid force field. 
To evaluate the performance of the OPC4 water model with all CHARMM36 simulation parameters (see Table S1 for full comparison), we compared the bulk water properties from the original OPC4 publication ${ }^{50}$ to simulation results obtained using the CHARMM36 parameter set. The comparison in Table 1 shows excellent agreement between the results obtained using the two parameter sets when the dispersion correction is used. The agreement is expected given that the bulk water properties should be largely unaffected by the cut-off distance, when the dispersion correction is employed. ${ }^{48}$ The results show that bulk water properties are well described in monolayer simulations with the CHARMM36 lipid model and the OPC4 water model, where the dispersion correction used. The bulk water properties without the dispersion correction, used in lipid bilayer simulations with the CHARMM36 force field, differ less than $1 \%$ from the data presented in the original OPC study. In addition to the bulk properties, the surface tension values of OPC4 water extracted from large systems (see Methods) simulated with the CHARMM36 parameter set $(70.5 \pm 0.4 \mathrm{mN} / \mathrm{m}$ at $298 \mathrm{~K}$ and $69.0 \pm 0.4 \mathrm{mN} / \mathrm{m}$ at $310 \mathrm{~K}$ ) were in good agreement with experimental values. Similar results for the OPC4 water model with the Slipids ${ }^{24,56}$ parameter set are provided in Section S2.1. Table 1: Comparison of selected key properties of bulk water between the values reported in the original OPC4 article $^{50}$ and the results obtained in this work with the CHARMM36 parameter set (with and without dispersion correction corresponding to suggested parameters for lipid monolayers and bilayers, respectively). The physical properties reported here are density $(\rho)$, self-diffusion coefficient $(D)$, location of the 1st peak in the oxygen-oxygen radial distribution function $r_{O O}$, and the thermal expansion coefficient $\alpha_{\mathrm{P}}$. The experimental values are shown in "Expt.". The simulations were carried out in a system comprised of 2228 water molecules at $298 \mathrm{~K}$. The use of the dispersion correction is denoted by "DC".

\begin{tabular}{lcccc}
\hline Property & Orig. OPC4 & OPC4/Charmm36 & OPC4$^{50}$ Charmm36 $^{\text {DC }}$ & Expt. \\
\hline$\rho\left(\mathrm{kg} / \mathrm{m}^{3}\right)$ & $997 \pm 1$ & $988 \pm 1$ & $997 \pm 1$ & 997 \\
$D\left(10^{-5} \mathrm{~cm}^{2} / \mathrm{s}\right)$ & $2.3 \pm 0.02$ & $2.20 \pm 0.02$ & $2.18 \pm 0.01$ & 2.3 \\
$1^{\text {st }}$ peak in $r_{O O}(\AA)$ & 2.80 & 2.80 & 2.80 & 2.80 \\
$\alpha_{\mathrm{P}}\left(10^{-4} \mathrm{~K}^{-1}\right)$ & $2.7 \pm 0.1$ & 2.68 & 2.80 & 2.56 \\
\hline
\end{tabular}

Since the used water model may drastically affect the properties of lipid bilayers in simulations, ${ }^{61}$ we verified that the CHARMM36 lipid force field used with the OPC4 water 
model gives appropriate lipid bilayer properties. APL values from simulation with the OPC4 water model are in good agreement with the results based on the CHARMM-specific TIP3P (TIPS3P) water model (see Table 2). DPPC bilayers seem to be somewhat too compressed with both water models, as recently observed, ${ }^{55}$ while results for POPC agree with experiments within error bars. Most importantly, the results obtained using the two water models are in mutual agreement. Furthermore, the two water models result in essentially identical density profiles of selected groups and acyl chain order parameters (Figs. S1 and S2). Similar conclusions can be drawn for average APL values, density profiles, and acyl chain order parameters calculated from simulations using the Slipids lipid force field together with the OPC4 water model, as discussed in Section S2.2.

Table 2: Average area per lipid values calculated for lipid bilayers. The standard deviation of the extracted values is shown. "Expt." stands for experimental data.

\begin{tabular}{lccc}
\hline Lipid & TIP(S)3P & OPC4 & Expt. $^{62}$ \\
\hline DPPC & $60.5 \pm 1.1$ & $59.3 \pm 1.3$ & $63.1 \pm 1.5$ \\
POPC & $63.7 \pm 1.1$ & $62.7 \pm 0.8$ & $64.3 \pm 1.3$ \\
\hline
\end{tabular}

Concluding, the results provide concrete evidence that the OPC4 water model can be used with the CHARMM36 force field, as well as with Slipids with their corresponding simulation parameter sets without perturbing the behavior of the lipids.

\section{Surface Pressure-Area Isotherms of Lipid Monolayers}

Surface pressure-area isotherms for DPPC and POPC monolayers at the air-water interface are shown in Fig. 2. The simulated isotherms are generally in good agreement with experiments considering the variation of isotherms reported in different experimental studies. The agreement is near-quantitative as significant differences occur only close to phase transitions and in the $\mathrm{L}_{\mathrm{C}}$ phase of the DPPC monolayers. Simulations from independent starting structures (Sets 1-3, see Methods) are in good agreement with each other for all systems.

Starting from POPC data for APL values between 60 and $110 \AA^{2}$, the POPC monolayers 
simulated with the CHARMM36 lipid and OPC4 water models are observed to reside in the $\mathrm{L}_{\mathrm{E}}$ phase (see Fig. S6), in good agreement with experimental data. The POPC systems remain stable also above the equilibrium collapse pressure $45 \mathrm{mN} / \mathrm{m}^{59,60}$ (with areas below $60 \AA^{2}$ ) because the simulation time and length scales are too short for monolayer rupture to take place. Slightly negative surface pressures are observed at APL values between 110 and $118 \AA^{2}$, while the values at 122 and $126 \AA^{2}$ is again zero within the error bars. The small negative surface pressures indicate a hysteresis-like metastability in the system, which would be released by a further phase separation into the gaseous phase. Indeed, there are clearly pores forming in the monolayer with an area per lipid of about $126 \AA^{2}$ (see Fig. S6), which might be a sign of phase coexistence with the gas phase. Finally, we note that POPC simulations with the CHARMM36 and Slipids lipid models combined with the OPC4 water model give consistent results with experiments for surface pressure-area isotherms at $310 \mathrm{~K}$ using smaller monolayer structures (see Fig. S8). Furthermore, in contrast to DPPC (see below), the isotherms for POPC at $298 \mathrm{~K}$ and $310 \mathrm{~K}$ are very similar in experiments and simulations (see Fig. S10).

DPPC monolayer expresses richer phase behavior than POPC. Fig. 2 highlights a clear transition to the $\mathrm{L}_{\mathrm{C}}$ phase and a region for $\mathrm{L}_{\mathrm{C}} / \mathrm{L}_{\mathrm{E}}$ phase coexistence for the DPPC monolayer. At $298 \mathrm{~K}$ the phase coexistence appears as a plateau in the surface pressure isotherm with a constant surface pressure value at $\sim 13 \mathrm{mN} / \mathrm{m}$ and APL values of $57-69 \AA^{2}$. Ordered and disordered domains are clearly detected in simulation snapshots taken from this plateau region, as demonstrated in Fig. 2. Voronoi tesselation analysis (see Methods) gave APL values of 56.1 and $68.5 \AA^{2}$ for $\mathrm{L}_{\mathrm{C}}$ and $\mathrm{L}_{\mathrm{E}}$ phases at the coexistence region, respectively. As expected, the values match the APL close to the edges of the coexistence plateau. The fractions of the more ordered $\mathrm{L}_{\mathrm{C}}$ phase $(15 \%, 38 \%, 58 \%, 77 \%$, and $97 \%)$ increase with decreasing area $\left(69,66,63,60\right.$, and $\left.57 \AA^{2}\right)$, respectively. Surface pressures just above the phase coexistence region (areas $72-78 \AA^{2}$ ) are slightly overestimated in different simulation sets, resembling a hysteresis effect, creating a shoulder in the isotherm that probably arises from incomplete 
Liquid condensed

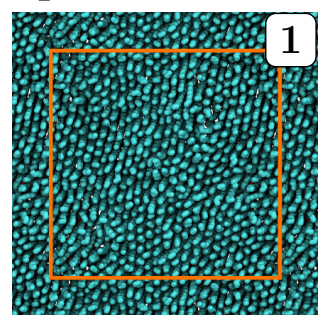

Liquid expanded
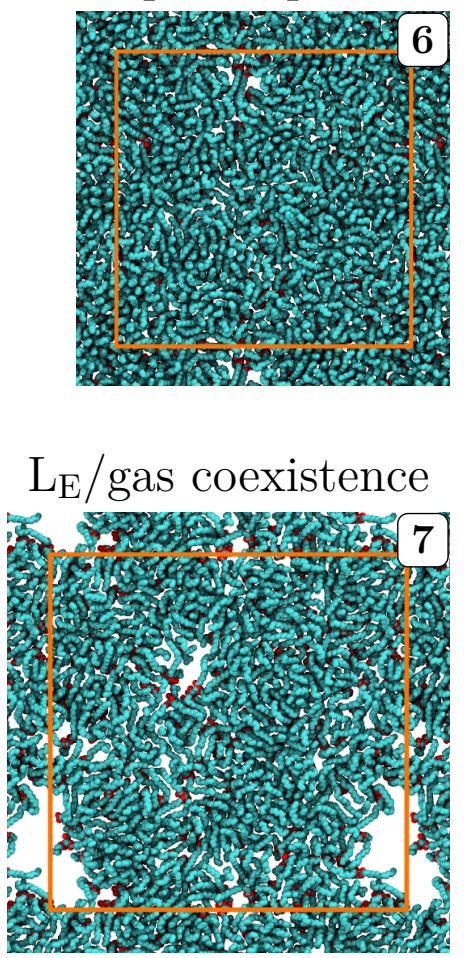
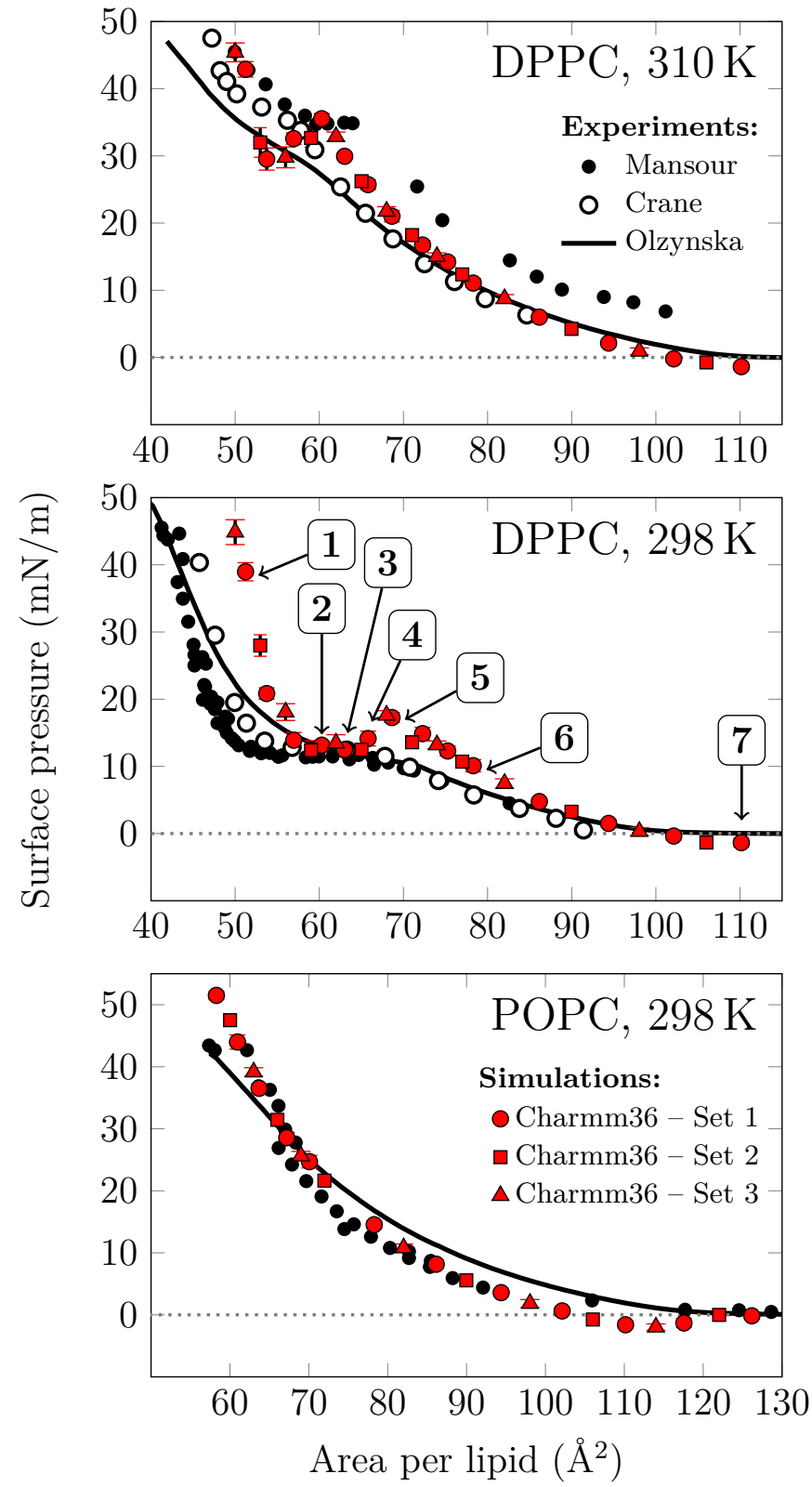

$\mathrm{L}_{\mathrm{C}} / \mathrm{L}_{\mathrm{E}}$ coexistence
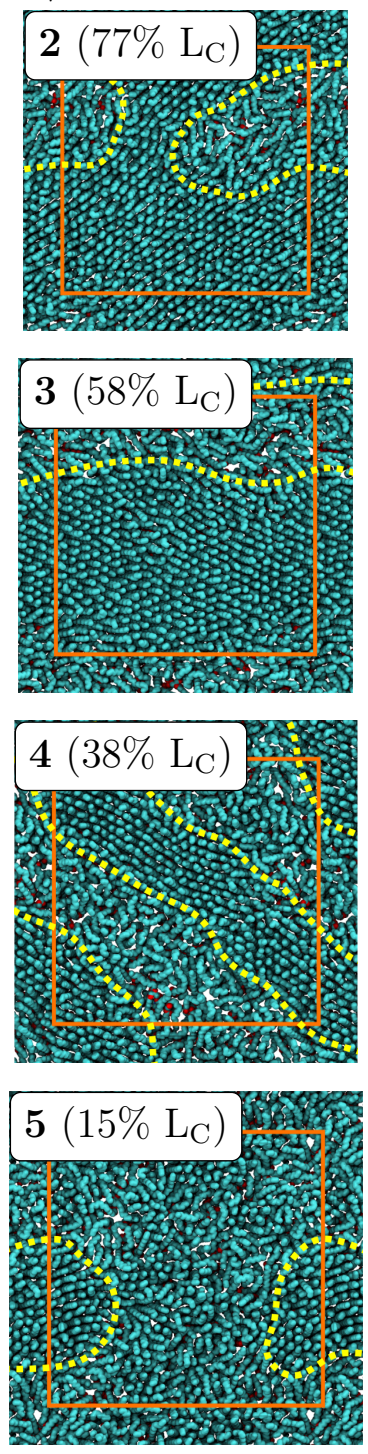

Figure 2: Surface pressure-area isotherms from simulations and experiments. The experimental isotherms are taken from refs. 60 (Mansour), 63 (Crane), and 34 (Olzynska). Selected snapshots of the DPPC monolayers at $298 \mathrm{~K}$ are shown to demonstrate the clearly visible phase coexistence region, which are surrounded by lines to guide the eye. Similar snapshots for DPPC at $310 \mathrm{~K}$ are shown in Fig. S7 and for POPC at $298 \mathrm{~K}$ in Fig. S6. Surface pressures were calculated from Eq. 2 by using surface tension values for the OPC4 water calculated from simulations $(69.1 \pm 2.0 \mathrm{mN} / \mathrm{m}$ at $298 \mathrm{~K}$ and $67.5 \pm 1.3 \mathrm{mN} / \mathrm{m}$ at $310 \mathrm{~K})$. 
equilibration close to the phase transition point. On the other hand, surface pressure is significantly overestimated with areas below $57 \AA^{2}$, where monolayer is in the $\mathrm{L}_{\mathrm{C}}$ phase. From the current data we cannot conclude if the discrepancy arises intrinsically from the lipid model itself, possibly having a transition to the $\mathrm{L}_{\mathrm{C}}$ phase with an average $\mathrm{APL}$ that is too large, or alternatively from an overestimated compressibility of the $\mathrm{L}_{\mathrm{C}}$ phase, possibly related to equilibration issues. The used CHARMM36 lipid model with the OPC4 water model resulted in the correct main transition temperature of the DPPC bilayer $(314 \mathrm{~K}$, details not shown), suggesting that the interactions are properly balanced to detect phase transitions at correct APL values. However, the differences between monolayer and bilayer behavior might not be properly captured by the force field. For example, different effects of the long-range LJ-potential in lipid bilayers and monolayers have been suggested due to the alkane-air interface. ${ }^{27,64}$

At $310 \mathrm{~K}$, the coexistence plateau in the DPPC system is less clear. In experimental data, a truly flat plateau is detected only when equilibration has been carried out carefully, ${ }^{60}$ while in experiments conducted with a constant compression rate ${ }^{34,63}$ there is only a change in the slope. In our simulations, a clear plateau is observed between 54-60 $\AA^{2}$ with a surface pressure around $31 \mathrm{mN} / \mathrm{m}$. This is in the same pressure range where slope changes were observed in experiments ${ }^{34,63}$ but slightly lower than in the most equilibrated experiments. ${ }^{60}$ Ordered and disordered domains in the plateau region are clearly visible in system snapshots (see Fig. S7) and the Voronoi tessellation analysis suggest APL values of 51.8 and $61.1 \AA^{2}$ for the $\mathrm{L}_{\mathrm{C}}$ and $\mathrm{L}_{\mathrm{E}}$ phases, respectively. These are in line with the APL values at the boundaries of the coexistence plateau. The fractions of the more ordered $\mathrm{L}_{\mathrm{C}}$ phase $(23 \%, 55 \%$, and $83 \%$ ) again increase with decreasing area $\left(60,57\right.$, and $\left.54 \AA^{2}\right)$, respectively.

Interestingly, pore formation is observed in all simulated systems around APL values of $110 \AA^{2}$, which coincides with the sudden drop in the nonlinear susceptibility observed in vibrational sum frequency generation (VSFG) experiments on DPPC monolayers. ${ }^{65}$ While this could be a coincidence, and the effect explained by an onset of gas and $\mathrm{L}_{\mathrm{E}}$ phase separation 
as discussed above, more careful analysis of this and the potential role of pore formation should be possible with the presented simulation model, which gives the correct energy cost for exposing the air-water interface. This is a significant advancement over previously used simulation models, where pore formation and phase separation occur at APL values that are much too small. ${ }^{20,21}$ Further analysis is, however, beyond the scope of the current work.

Significant finite size effects were observed only in simulations where phase coexistence was present. Experimental isotherms are reproduced in simulations for POPC monolayers, where phase coexistence was not observed, also with smaller simulation system sizes when the CHARMM36 lipid model was used with the OPC4 water model, as seen in Fig. S8. On the other hand, simulations with smaller DPPC monolayer systems at $310 \mathrm{~K}$ do not capture the $\mathrm{L}_{\mathrm{C}} / \mathrm{L}_{\mathrm{E}}$ coexistence region and show significant deviations from experimental results as illustrated in Fig. S8.

Nonetheless, in all cases the added value of the OPC4 model to describe the surface tension in a realistic manner is evident. This is exemplified by comparison between simulations with smaller system sizes in Fig. S8, which clearly demonstrates the improvement in surface pressure-area isotherms also for the POPC monolayer, when the OPC4 water model is used. The improvement is significant for both the CHARMM36 and the Slipids lipid models.

\section{Conclusions}

We have shown that the recently developed 4 -point OPC water model ${ }^{50}$ is able to reproduce the surface tension of water with LJ cut-off lengths that are compatible with the CHARMM $36^{27}$ and Slipids ${ }^{24,56}$ force fields. Furthermore, the combination of OPC4 with CHARMM36 reproduces experimental data of the surface pressure-area isotherm in single-component DPPC and POPC monolayers with almost quantitative accuracy. Minor discrepancies occur only close to phase transition boundaries and in the $\mathrm{L}_{\mathrm{C}}$ phase. The former can be explained by limited sampling times in simulations, while the latter may be related to the quality of the 
used lipid force field. Importantly, relatively large systems are needed to simulate ordered and phase coexistence regions, while smaller systems are adequate for considerations of disordered single-phase regions.

The most striking result of the present work is the ability of the OPC4/CHARMM36 combination to describe the surface pressure-area isotherm in near-quantitative accuracy even in phase coexistence regions. The approach introduced here is, to our knowledge, the only atomistic-resolution simulation model for lipid monolayers able to do so without any obvious simulation artefacts. ${ }^{33}$

It is possible that the present approach of combining OPC4 with CHARMM36 has a number of promising applications in related contexts. The most obvious candidates include

atomistic simulations of pulmonary surfactant and tear film lipid layers, ${ }^{7,11,12}$ both having an important role in our health, and the numerous materials science applications exploiting monolayer structures.

\section{Acknowledgement}

We thank CSC - IT Center for Science (Espoo, Finland) for computing resources. MJ and IV thank the Academy of Finland (Centre of Excellence project (grant no. 307415)) and the European Research Council (Advanced Grant project CROWDED-PRO-LIPIDS (grant no. 290974)) for financial support. LC acknowledges the support from the Czech Science Foundation (grant no. 17-06792S).

\section{Supporting Information Available}

Details on additional simulations and additional results. 


\section{References}

(1) Rugonyi, S.; Biswas, S.; Hall, S. The Biophysical Function of Pulmonary Surfactant. Respir. Physiol. Neurobiol. 2008, 163, 244-255.

(2) Rantamäki, A. H.; Telenius, J.; Koivuniemi, A.; Vattulainen, I.; Holopainen, J. M. Lessons From the Biophysics of Interfaces: Lung Surfactant and Tear Fluid. Prog. Retin. Eye Res. 2011, 30, 204-215.

(3) Calvez, P.; Bussières, S.; Éric Demers,; Salesse, C. Parameters Modulating the Maximum Insertion Pressure of Proteins and Peptides in Lipid Monolayers. Biochimie 2009, 91, $718-733$.

(4) Kaganer, V. M.; Möhwald, H.; Dutta, P. Structure and Phase Transitions in Langmuir Monolayer. Rev. Mod. Phys. 1999, 71, 779-819.

(5) Duncan, S. L.; Larson, R. G. Comparing Experimental and Simulated Pressure-Area Isotherms for DPPC. Biophys. J. 2008, 94, 2965-2986.

(6) Baoukina, S.; Tieleman, D. P. Biomolecular Simulations: Methods and Protocols; Humana Press: Totowa, NJ, 2013; pp 431-444.

(7) Baoukina, S.; Tieleman, D. P. Computer Simulations of Lung Surfactant. BBABiomembranes 2016, 1858, 2431-2440.

(8) Kulovesi, P.; Telenius, J.; Koivuniemi, A.; Brezesinski, G.; Rantamaki, A.; Viitala, T.; Puukilainen, E.; Ritala, M.; Wiedmer, S. K.; Vattulainen, I.; Holopainen, J. M. Molecular Organization of the Tear Fluid Lipid Layer. Biophys. J. 2010, 99, 2559-2567.

(9) Kulovesi, P.; Telenius, J.; Koivuniemi, A.; Brezesinski, G.; Vattulainen, I.; Holopainen, J. M. The Impact of Lipid Composition on the Stability of the Tear Fluid Lipid Layer. Soft Matter 2012, 8, 5826-5834. 
(10) Rantamaki, A.; Javanainen, M.; Vattulainen, I.; Holopainen, J. M. Do Lipids Retard the Evaporation of the Tear Fluid? Investigative Ophthalmology \& Visual Science 2012, 53, 6442-6447.

(11) Telenius, J.; Koivuniemi, A.; Kulovesi, P.; Holopainen, J. M.; Vattulainen, I. Role of Neutral Lipids in Tear Fluid Lipid Layer: Coarse-Grained Simulation Study. Langmuir 2012, 28, 17092-17100.

(12) Cwiklik, L. Tear Film Lipid Layer: A Molecular Level View. BBA-Biomembranes 2016, $1858,2421-2430$.

(13) Baoukina, S.; Monticelli, L.; Marrink, S. J.; Tieleman, D. P. Pressure-Area Isotherm of a Lipid Monolayer From Molecular Dynamics Simulations. Langmuir 2007, 23, $12617-12623$.

(14) Javanainen, M.; Monticelli, L.; de la Serna, J. B.; Vattulainen, I. Free Volume Theory Applied to Lateral Diffusion in Langmuir Monolayers: Atomistic Simulations for a Protein-Free Model of Lung Surfactant. Langmuir 2010, 26, 15436-15444.

(15) Javanainen, M.; Hammaren, H.; Monticelli, L.; Jeon, J.-H.; Miettinen, M. S.; MartinezSeara, H.; Metzler, R.; Vattulainen, I. Anomalous and Normal Diffusion of Proteins and Lipids in Crowded Lipid Membranes. Farad. Discuss. 2013, 161, 397-417.

(16) Baoukina, S.; Mendez-Villuendas, E.; Tieleman, D. P. Molecular View of Phase Coexistence in Lipid Monolayers. J. Am. Chem. Soc. 2012, 134, 17543-17553.

(17) Baoukina, S.; Monticelli, L.; Amrein, M.; Tieleman, D. P. The Molecular Mechanism of Monolayer-Bilayer Transformations of Lung Surfactant From Molecular Dynamics Simulations. Biophys. J. 2007, 93, 3775-3782.

(18) Baoukina, S.; Monticelli, L.; Risselada, H. J.; Marrink, S. J.; Tieleman, D. P. The 
Molecular Mechanism of Lipid Monolayer Collapse. Proc. Natl. Acad. Sci. USA 2008, $105,10803-10808$.

(19) Baoukina, S.; Rozmanov, D.; Mendez-Villuendas, E.; Tieleman, D. P. The Mechanism of Collapse of Heterogeneous Lipid Monolayers. Biophys. J. 2014, 107, 1136-1145.

(20) Knecht, V.; Müller, M.; Bonn, M.; Marrink, S.-J.; Mark, A. E. Simulation Studies of Pore and Domain Formation in a Phospholipid Monolayer. The Journal of Chemical Physics 2005, 122, 024704.

(21) Nisoh, N.; Karttunen, M.; Monticelli, L.; Wong-ekkabut, J. Lipid Monolayer Disruption Caused by Aggregated Carbon Nanoparticles. RSC Adv. 2015, 5, 11676-11685.

(22) Baoukina, S.; Tieleman, D. P. Lung Surfactant Protein SP-B Promotes Formation of Bilayer Reservoirs From Monolayer and Lipid Transfer Between the Interface and Subphase. Biophys. J. 2011, 100, $1678-1687$.

(23) Barnoud, J.; Urbini, L.; Monticelli, L. C60 Fullerene Promotes Lung Monolayer Collapse. J. R. Soc. Interface 2015, 12, 20140931.

(24) Jämbeck, J. P.; Lyubartsev, A. P. Derivation and Systematic Validation of a Refined All-Atom Force Field for Phosphatidylcholine Lipids. J. Phys. Chem. B 2012, 116, $3164-3179$.

(25) Maciejewski, A.; Pasenkiewicz-Gierula, M.; Cramariuc, O.; Vattulainen, I.; Rog, T. Refined OPLS All-Atom Force Field for Saturated Phosphatidylcholine Bilayers at Full Hydration. J. Phys. Chem. B 2014, 118, 4571-4581.

(26) Dickson, C. J.; Madej, B. D.; Skjevik, A. A.; Betz, R. M.; Teigen, K.; Gould, I. R.; Walker, R. C. Lipid14: The Amber Lipid Force Field. J. Chem. Theory Comput. 2014, $10,865-879$. 
(27) Klauda, J. B.; Venable, R. M.; Freites, J. A.; O'Connor, J. W.; Tobias, D. J.; MondragonRamirez, C.; Vorobyov, I.; MacKerell Jr, A. D.; Pastor, R. W. Update of the CHARMM All-Atom Additive Force Field for Lipids: Validation on Six Lipid Types. J. Phys. Chem. $B$ 2010, 114, 7830-7843.

(28) Jorgensen, W. L.; Chandrasekhar, J.; Madura, J. D.; Impey, R. W.; Klein, M. L. Comparison of Simple Potential Functions for Simulating Liquid Water. J. Chem. Phys. 1983, 79, 926-935.

(29) Vega, C.; De Miguel, E. Surface Tension of the Most Popular Models of Water by Using the Test-Area Simulation Method. J. Chem. Phys. 2007, 126, 154707.

(30) Chen, F.; Smith, P. E. Simulated Surface Tensions of Common Water Models. J. Chem. Phys. 2007, 126, 221101.

(31) Vargaftik, N.; Volkov, B.; Voljak, L. International Tables of the Surface Tension of Water. J. Phys. Chem. Ref. Data 1983, 12, 817-820.

(32) Marrink, S. J.; Risselada, H. J.; Yefimov, S.; Tieleman, D. P.; De Vries, A. H. The MARTINI Force Field: Coarse Grained Model for Biomolecular Simulations. J. Phys. Chem. B 2007, 111, 7812-7824.

(33) Lamberg, A.; Ollila, O. S. Comment on "Structural Properties of POPC Monolayers Under Lateral Compression: Computer Simulations Analysis". Langmuir 2015, 31, 886-887.

(34) Olżyńska, A.; Zubek, M.; Roeselova, M.; Korchowiec, J.; Cwiklik, L. Mixed DPPC/POPC Monolayers: All-Atom Molecular Dynamics Simulations and Langmuir Monolayer Experiments. BBA-Biomembranes 2016, 1858, 3120-3130.

(35) Rose, D.; Rendell, J.; Lee, D.; Nag, K.; Booth, V. Molecular Dynamics Simulations of Lung Surfactant Lipid Monolayers. Biophys. Chem. 2008, 138, 67-77. 
(36) Mohammad-Aghaie, D.; Macé, E.; Sennoga, C. A.; Seddon, J. M.; Bresme, F. Molecular Dynamics Simulations of Liquid Condensed to Liquid Expanded Transitions in DPPC Monolayers. J. Phys. Chem. B 2009, 114, 1325-1335.

(37) Mohammad-Aghaie, D.; Bresme, F. Force-Field Dependence on the Liquid-Expanded to Liquid-Condensed Transition in DPPC Monolayers. Mol. Simul. 2016, 42, 391-397.

(38) Lamberg, A.; Taniguchi, T. Coarse-grained computational studies of supported bilayers: current problems and their root causes. The Journal of Physical Chemistry B 2014, $118,10643-10652$.

(39) Shinoda, W.; DeVane, R.; Klein, M. L. Zwitterionic Lipid Assemblies: Molecular Dynamics Studies of Monolayers, Bilayers, and Vesicles Using a New Coarse Grain Force Field. J. Phys. Chem. B 2010, 114, 6836-6849.

(40) Hu, H.; Wang, F. The Liquid-Vapor Equilibria of TIP4P/2005 and BLYPSP-4F Water Models Determined Through Direct Simulations of the Liquid-Vapor Interface. J. Chem. Phys. 2015, 142, 214507.

(41) Abascal, J. L.; Vega, C. A General Purpose Model for the Condensed Phases of Water: TIP4P/2005. J. Chem. Phys. 2005, 123, 234505.

(42) Ismail, A. E.; Grest, G. S.; Stevens, M. J. Capillary Waves at the Liquid-Vapor Interface and the Surface Tension of Water. J. Chem. Phys. 2006, 125, 014702.

(43) Essmann, U.; Perera, L.; Berkowitz, M. L.; Darden, T.; Lee, H.; Pedersen, L. G. A Smooth Particle Mesh Ewald Method. J. Chem. Phys. 1995, 103, 8577-8593.

(44) Darden, T.; York, D.; Pedersen, L. Particle Mesh Ewald: An N. $\log (\mathrm{N})$ Method for Ewald Sums in Large Systems. J. Chem. Phys. 1993, 98, 10089-10092.

(45) Alejandre, J.; Chapela, G. A. The Surface Tension of TIP4P/2005 Water Model Using the Ewald Sums for the Dispersion Interactions. J. Chem. Phys. 2010, 132, 014701. 
(46) Huang, K.; García, A. E. Effects of Truncating van der Waals Interactions in Lipid Bilayer Simulations. J. Chem. Phys. 2014, 141, 105101.

(47) Wennberg, C. L.; Murtola, T.; Hess, B.; Lindahl, E. Lennard-Jones Lattice Summation in Bilayer Simulations Has Critical Effects on Surface Tension and Lipid Properties. J. Chem. Theory Comput. 2013, 9, 3527-3537.

(48) Shirts, M. R.; Mobley, D. L.; Chodera, J. D.; Pande, V. S. Accurate and Efficient Corrections for Missing Dispersion Interactions in Molecular Simulations. J. Phys. Chem. B 2007, 111, 13052-13063.

(49) Lundberg, L.; Edholm, O. Dispersion Corrections to the Surface Tension at Planar Surfaces. J. Chem. Theory Comput. 2016, 12, 4025-4032.

(50) Izadi, S.; Anandakrishnan, R.; Onufriev, A. V. Building Water Models: A Different Approach. J. Phys. Chem. Lett. 2014, 5, 3863-3871.

(51) Izadi, S.; Onufriev, A. V. Accuracy Limit of Rigid 3-Point Water Models. J. Chem. Phys. 2016, 145, 074501.

(52) in 't Veld, P. J.; Ismail, A. E.; Grest, G. S. Application of Ewald Summations to Long-Range Dispersion Forces. J. Chem. Phys. 2007, 127, 144711.

(53) Abraham, M. J.; Murtola, T.; Schulz, R.; Páll, S.; Smith, J. C.; Hess, B.; Lindahl, E. GROMACS: High Performance Molecular Simulations Through Multi-Level Parallelism From Laptops to Supercomputers. SoftwareX 2015, 1, 19-25.

(54) Pronk, S.; Páll, S.; Schulz, R.; Larsson, P.; Bjelkmar, P.; Apostolov, R.; Shirts, M. R.; Smith, J. C.; Kasson, P. M.; van der Spoel, D. GROMACS 4.5: A High-Throughput and Highly Parallel Open Source Molecular Simulation Toolkit. Bioinformatics 2013, btt055. 
(55) Lee, J. et al. CHARMM-GUI Input Generator for NAMD, GROMACS, AMBER, OpenMM, and CHARMM/OpenMM Simulations Using the CHARMM36 Additive Force Field. J. Chem. Theory Comput. 2015, 12, 405-413.

(56) Jämbeck, J. P.; Lyubartsev, A. P. An Extension and Further Validation of an AllAtomistic Force Field for Biological Membranes. J. Chem. Theory Comput. 2012, 8, 2938-2948.

(57) Tribello, G. A.; Bonomi, M.; Branduardi, D.; Camilloni, C.; Bussi, G. PLUMED 2: New Feathers for an Old Bird. Comput. Phys. Commun. 2014, 185, 604-613.

(58) Guixà-González, R.; Rodriguez-Espigares, I.; Ramírez-Anguita, J. M.; Carrió-Gaspar, P.; Martinez-Seara, H.; Giorgino, T.; Selent, J. MEMBPLUGIN: Studying Membrane Complexity in VMD. Bioinformatics 2014, 30, 1478-1480.

(59) Lee, S.; Kim, D. H.; Needham, D. Equilibrium and Dynamic Interfacial Tension Measurements at Microscopic Interfaces Using a Micropipet Technique. 2. Dynamics of Phospholipid Monolayer Formation and Equilibrium Tensions at the Water-Air Interface. Langmuir 2001, 17, 5544-5550.

(60) Mansour, H. M.; Zografi, G. Relationships Between Equilibrium Spreading Pressure and Phase Equilibria of Phospholipid Bilayers and Monolayers at the Air-Water Interface. Langmuir 2007, 23, 3809-3819.

(61) Piggot, T. J.; Piñeiro, A.; Khalid, S. Molecular Dynamics Simulations of Phosphatidylcholine Membranes: A Comparative Force Field Study. J. Chem. Theory Comput. 2012, 8, 4593-4609.

(62) Kučerka, N.; Nieh, M.-P.; Katsaras, J. Fluid Phase Lipid Areas and Bilayer Thicknesses of Commonly Used Phosphatidylcholines as a Function of Temperature. BBABiomembranes 2011, 1808, 2761-2771. 
(63) Crane, J. M.; Putz, G.; Hall, S. B. Persistence of Phase Coexistence in Disaturated Phosphatidylcholine Monolayers at High Surface Pressures. Biophys. J. 1999, 77, 31343143.

(64) Klauda, J. B.; Wu, X.; Pastor, R. W.; Brooks, B. R. Long-Range Lennard-Jones and Electrostatic Interactions in Interfaces: Application of the Isotropic Periodic Sum Method. J. Phys. Chem. B 2007, 111, 4393-4400.

(65) Roke, S.; Schins, J.; Müller, M.; Bonn, M. Vibrational Spectroscopic Investigation of the Phase Diagram of a Biomimetic Lipid Monolayer. Phys. Rev. Lett. 2003, 90, 128101. 


\section{Graphical TOC Entry}

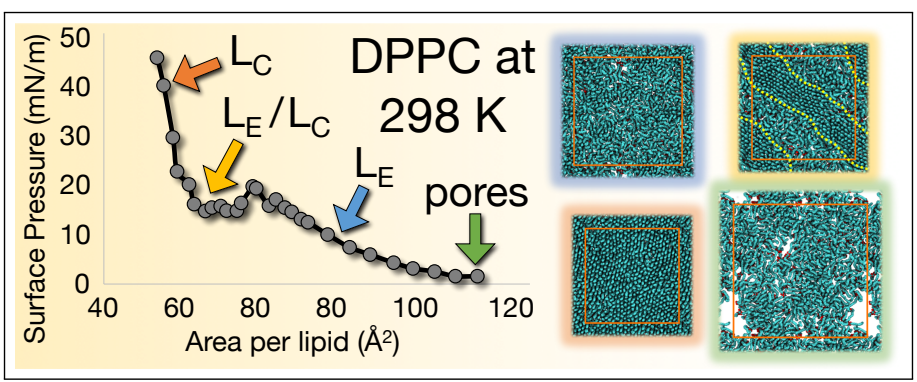

